\title{
TOTAL ANGULAR DEFECT AND EULER'S THEOREM FOR POLYHEDRA
}

\author{
Dong-SoO KIM ${ }^{\mathrm{a}, *}$ AND Young Ho KIM ${ }^{\mathrm{b}}$
}

\begin{abstract}
We give an elementary proof of Descartes' theorem for polyhedra. Since Descartes' theorem is equivalent to Euler's theorem for polyhedra, this also gives an elementary proof of Euler's theorem.
\end{abstract}

\section{INTRODUCTION}

Let us begin with a convex polyhedron $\Sigma$. Euclid proved that the sum of the face angles at any vertex $P$ of $\Sigma$ is less than $2 \pi$; the difference between this sum and $2 \pi$ is called the angular defect at $P$ and denoted by $\Delta_{\Sigma}(P)$. If we sum the angular defects over all the vertices of $\Sigma$, we obtain the total angular defect $\Delta(\Sigma)$ ([3] or [4]). René Descartes used spherical trigonometry to prove that $\Delta(\Sigma)=4 \pi$ for every convex polyhedron $\Sigma$. Descartes' theorem for polyhedra in space is analogous to the exterior angle theorem for polygons in a plane ([5]). A number of proofs of the exterior angle theorem are given in [2].

For a convex polygon $\sigma$ we denote by $\delta_{\sigma}(P)$ and $\delta(\sigma)$ the exterior angle of $\sigma$ at a vertex $P$ of $\sigma$ and the sum of exterior angles of $\sigma$, respectively. Then we give a proof of the exterior angle theorem for convex polygons as follows.

A proof of the exterior angle theorem. First note the following which can be checked easily:

$\left(L_{1}\right)$ Every triangle $\sigma$ has total exterior angle $\delta(\sigma)=2 \pi$.

$\left(L_{2}\right)$ If a convex polygon $\sigma$ is dissected by a straight line $l$ into two polygons $\sigma_{1}$ and $\sigma_{2}$, then we have the following:

Received by the editors September 1, 2011. Accepted February 10, 2012.

2000 Mathematics Subject Classification. 52B05, 53B45.

Key words and phrases. polygon, polyhedron, exterior angle theorem, Euler's theorem, Descartes' theorem.

${ }^{*}$ Corresponding author. 
Let $P_{1} P_{2}$ denote the common edge of $\sigma_{1}$ and $\sigma_{2}$. Then for each $i=1,2$ we have

$$
\delta_{\sigma_{1}}\left(P_{i}\right)+\delta_{\sigma_{2}}\left(P_{i}\right)=\delta_{\sigma}\left(P_{i}\right)+\pi,
$$

and hence we get

$$
\delta\left(\sigma_{1}\right)+\delta\left(\sigma_{2}\right)=\delta(\sigma)+2 \pi
$$

$\left(L_{3}\right)$ Let $\sigma$ be a convex polygon which is not a triangle. Then $\sigma$ can be dissected by a straight line into two polygons $\sigma_{1}$ and $\sigma_{2}$ which have less number of edges than that of the polygon $\sigma$.

Then, together with $\left(L_{1}\right),\left(L_{2}\right)$, and $\left(L_{3}\right)$, a mathematical induction argument on the number of edges of convex polygons shows the exterior angle theorem for convex polygons.

Hence it is quite natural to consider whether a similar argument can be made on the total angular defect of convex polyhedra.

In this short note, we give an affirmative answer, that is, we give a proof of Descartes' theorem for convex polyhedra as that of an exterior angle theorem for polygons.

First of all, we give a proposition which shows that Descartes' theorem is equivalent to Euler's theorem for convex polyhedra ([3] or [4]).

Proposition 1. For a convex polyhedron $\Sigma$, we have $\Delta(\Sigma)=2 \pi(V-E+F)$, where $V, E$, and $F$ denote the number of vertices, edges, and faces, respectively.

Proof. We give a proof for completeness. For each vertex $P$ of $\Sigma$, we put $r=r(P)$ as the number of edges which are incident with $P$. If we denote by $\alpha_{1}, \cdots, \alpha_{r}$ the face angles at $P$, then the angular defect $\Delta(P)$ at $P$ is

$$
\begin{aligned}
\Delta(P) & =2 \pi-\left(\alpha_{1}+\cdots+\alpha_{r}\right) \\
& =\left(\pi-\alpha_{1}\right)+\cdots+\left(\pi-\alpha_{r}\right)-r \pi+2 \pi .
\end{aligned}
$$

Hence the total angular defect of $\Sigma$ is given by

$$
\Delta(\Sigma)=\sum_{P}\left\{\left(\pi-\alpha_{1}\right)+\cdots+\left(\pi-\alpha_{r}\right)\right\}-\pi \sum_{P} r(P)+2 \pi \sum_{P} 1,
$$

where the summations are taken over all vertices $P$ of $\Sigma$. Note that the first sum is nothing but the sum of exterior angles of all plane face angles of the polyhedron $\Sigma$, and hence it simply becomes $2 \pi F$ by the exterior angle theorem. Obviously, we 
have

$$
\sum_{P} 1=V
$$

Furthermore, since every edge has exactly two vertices, we also obtain

$$
\sum_{P} r(P)=2 E \text {. }
$$

Thus, together with (4) and (5), (3) completes the proof.

\section{Proof of the Main Theorem}

Now we establish formulas for total angular defect of polyhedra which are analogous to the formulas (1) and (2) for the total exterior angle of polygons.

Lemma 2. Let $\Sigma$ be a convex polyhedron which can be dissected by a plane $\varphi$ into two convex polyhedra $\Sigma_{1}$ and $\Sigma_{2}$. Then we have the following:

Let $\sigma$ be a convex $r$-sided polygon which is the common face of $\Sigma_{1}$ and $\Sigma_{2}$ with vertices $P_{1}, \cdots, P_{r}$. Then for each $i=1,2, \cdots, r$ we have

$$
\Delta_{\Sigma_{1}}\left(P_{i}\right)+\Delta_{\Sigma_{2}}\left(P_{i}\right)=\Delta_{\Sigma}\left(P_{i}\right)+2 \delta_{\sigma}\left(P_{i}\right),
$$

and we get

$$
\Delta\left(\Sigma_{1}\right)+\Delta\left(\Sigma_{2}\right)=\Delta(\Sigma)+4 \pi .
$$

Proof. Note that if $P_{i}$ is not a vertex of $\Sigma$, then we have $\Delta_{\Sigma}\left(P_{i}\right)=0$. For simplicity, we prove (6) when $P_{i}$ is a vertex of $\Sigma$ and every edge of $\sigma$ is an edge of $\Sigma$. The remaining cases can be treated similarly. We denote by $\gamma_{i}$ the interior angle of $\sigma$ at $P_{i}, i=1, \cdots, r$.

Let $\alpha_{i j}$ and $\beta_{i k}$ denote the face angles of $\Sigma$ at $P_{i}$ which are face angles of $\Sigma_{1}$ and $\Sigma_{2}$, respectively. Then, at each $P_{i}$, we have the following:

$$
\begin{gathered}
\Delta_{\Sigma}\left(P_{i}\right)=2 \pi-\sum_{j} \alpha_{i j}-\sum_{k} \beta_{i k}, \\
\Delta_{\Sigma_{1}}\left(P_{i}\right)=2 \pi-\sum_{j} \alpha_{i j}-\gamma_{i},
\end{gathered}
$$

and

$$
\Delta_{\Sigma_{2}}\left(P_{i}\right)=2 \pi-\sum_{k} \beta_{i k}-\gamma_{i}
$$

Thus it follows from (8), (9) and (10) directly that (6) holds. 
Let's denote by $\Delta_{1}$ and $\Delta_{2}$ the sum of angular defects at vertices of $\Sigma_{1}$ and $\Sigma_{2}$, respectively, which do not belong to the plane $\varphi$.

Then we obtain from (6) and the exterior angle theorem that

$$
\begin{aligned}
\Delta\left(\Sigma_{1}\right)+\Delta\left(\Sigma_{2}\right) & =\Delta_{1}+\Delta_{2}+\sum_{i}\left\{\Delta_{\Sigma_{1}}\left(P_{i}\right)+\Delta_{\Sigma_{2}}\left(P_{i}\right)\right\} \\
& =\Delta_{1}+\Delta_{2}+\sum_{i} \Delta_{\Sigma}\left(P_{i}\right)+2 \sum_{i} \delta_{\sigma}\left(P_{i}\right) \\
& =\Delta(\Sigma)+4 \pi,
\end{aligned}
$$

which completes the proof.

Next, we prove a lemma for convex polyhedra which is analogous to $\left(L_{3}\right)$ for convex polygons.

Lemma 3. Let $\Sigma$ be a convex polyhedron which is not a tetrahedron. Then $\Sigma$ can be dissected by planes successively into a finite number of polyhedra $\Sigma_{1}, \cdots, \Sigma_{m}$ such that each $\Sigma_{i}$ has less faces than those of $\Sigma$.

Proof. Recall that for a vertex $P$ of $\Sigma, r(P)(\geq 3)$ denotes the number of edges of $\Sigma$ which are incident with $P$. First of all, we treat two cases.

Case 1. Suppose $r(P) \geq 4$ for some vertex $P$ of $\Sigma$. Then choose two vertices $P_{1}$ and $P_{2}$ such that the segments $P P_{1}$ and $P P_{2}$ are the edges of $\Sigma$ which are not those of a common face of $\Sigma$. Then the plane through $P, P_{1}$, and $P_{2}$ dissects $\Sigma$ into two polyhedra $\Sigma_{1}$ and $\Sigma_{2}$ which have the desired property.

Case 2. Suppose $\Sigma$ has a triangular face $P_{1} P_{2} P_{3}$. By Case 1 , we may assume that $r(P)=3$ for every vertex $P$ of $\Sigma$. Choose a vertex $P_{4}$ of $\Sigma$ which forms an edge $P_{1} P_{4}$ of $\Sigma$ together with $P_{1}$ other than $P_{1} P_{2}$ and $P_{1} P_{3}$. Then by the plane through $P_{2}, P_{3}$ and $P_{4}, \Sigma$ is dissected into a tetrahedron $\Sigma_{1}$ and a convex polyhedron $\Sigma_{2}$. Note that $\Sigma_{2}$ has the same number of faces as that of $\Sigma$. Furthermore, $\Sigma_{2}$ satisfies $r_{\Sigma_{2}}\left(P_{4}\right)=4$. Hence Case 1 shows that $\Sigma_{2}$ can be dissected by a plane into two convex polyhedra $\Sigma_{3}$ and $\Sigma_{4}$. Eventually, $\Sigma$ can be dissected by planes successively into 3 polyhedra $\Sigma_{1}, \Sigma_{3}, \Sigma_{4}$ all of which have the desired property.

Now we denote by $n(\Sigma)$ the minimum number of edges for all faces of $\Sigma$. Then we prove Lemma 3 by a mathematical induction on $n=n(\Sigma)$. If $n(\Sigma)=3$, then Case 2 implies Lemma 3. Suppose that Lemma 3 holds for all convex polyhedra $\Sigma$ with $n(\Sigma) \leq k, k \geq 3$. If $\Sigma$ is a convex polyhedron with $n(\Sigma)=k+1$, then we consider a convex $(k+1)$-gonal face $P_{1} P_{2} \cdots P_{k+1}$ of $\Sigma$. By Case 1 , we may assume that $r(P)=3$ for every vertex $P$ of $\Sigma$. Choose a vertex $Q$ of $\Sigma$ which forms an 
edge $P_{k} Q$ of $\Sigma$ together with $P_{k}$ other than $P_{k-1} P_{k}$ and $P_{k} P_{k+1}$. Then the plane through $P_{1}, P_{k}$, and $Q$ dissects $\Sigma$ into two polyhedra $\Sigma_{1}$ and $\Sigma_{2}$, which have faces, respectively, less than or equal to the number of faces of $\Sigma$. Obviously, we have $n\left(\Sigma_{1}\right) \leq k$, and $n\left(\Sigma_{2}\right) \leq k$. Hence the induction hypothesis completes the proof of Lemma 3 .

Finally, we prove Descartes' theorem for convex polyhedra.

Theorem 4. Let $\Sigma$ be a convex polyhedron. Then the total angular defect is given by $\Delta(\Sigma)=4 \pi$.

Proof. We prove by a mathematical induction on the number of faces $F=F(\Sigma)$. If $F(\Sigma)=4$, that is, $\Sigma$ is a tetrahedron, then it is obvious that the total angular defect of $\Sigma$ is $4 \pi$. Suppose that the theorem holds for all convex polyhedra $\Sigma$ with $F(\Sigma) \leq k, k \geq 4$. If $\Sigma$ is a convex polyhedron with $F(\Sigma)=k+1$, then Lemma 3 shows that $\Sigma$ can be dissected by planes successively into a finite number of convex polyhedra $\Sigma_{1}, \Sigma_{2}, \cdots, \Sigma_{m}$ such that $F\left(\Sigma_{i}\right) \leq k$. Hence from the induction hypothesis we see that each $\Sigma_{i}$ satisfies $\Delta\left(\Sigma_{i}\right)=4 \pi$. Thus, by repeatedly using Lemma 2 , we conclude that $\Delta(\Sigma)=4 \pi$.

As a corollary of Lemma 3, we give a dissection theorem for polyhedra as follows (cf. [1, p. 212]).

Corollary 5. Every polyhedron can be dissected by planes successively into a finite number of tetrahedra.

Proof. We may use the same induction argument as above to prove Corollary 5 for a convex polyhedron. For an arbitrary polyhedron $\Sigma$, let $\varphi_{1}, \cdots, \varphi_{m}$ denote the face planes. Then $\varphi_{1}, \cdots, \varphi_{m}$ divide space into a set of convex regions, a finite number of which consist of the polyhedron $\Sigma$. Hence the polyhedron $\Sigma$ can be dissected by planes successively into a finite number of convex polyhedron. Thus the corollary follows from the convex case.

\section{REFERENCES}

1. Eves, H.: A Survey of Geometry, Revised Edition. Allyn and Bacon, Inc., Boston, 1972.

2. Federico, P. J.: Descartes on Polyhedra: A Study of the De Solidorum Elementis. Springer, New York, 1982.

3. Hilton, P. \& Pedersen, J.: Descartes, Euler, Poincaré, Pólya. Enseign. Math. 27 (1981), 327-343. 
4. ___ The Euler Characteristic and Polya's Dream. Amer. Math. Monthly 103 (1996), 121-131.

5. Polya, G.: Mathematical Discovery, Combined Edition. John Wiley and Sons, Inc., New York, 1981.

${ }^{a}$ Department of Mathematics, Chonnam National University, Kwanguu 500-757, Korea Email address: dosokim@chonnam.ac.kr

${ }^{\text {b}}$ Department of Mathematics, College of Natural Sciences, Kyungpook National UniVERSITY, TAEGU, 702-701, KorEA

Email address: yhkim@knu.ac.kr 